\title{
A Robot for Combat and Calamities with Encrypted WSN
}

\author{
S Abiraj ${ }^{\text {a,1 }}$, D Eeswar Samhithan ", B Sanjai Kumar ${ }^{\text {a }}$, Y Venkatesh ${ }^{\text {a }}$ \\ ${ }^{a}$ Department of Electronics and Communication Engineering Vel Tech High Tech Dr \\ Rangarajan Dr Sakunthala Engineering College, Chennai, TN, India
}

\begin{abstract}
The needs of search and rescue teams in numerous ways reflect the requirements of the military. They both work in perilous situations, they need to discover ways to gather data while keeping personnel out of hurt, and they are both seeking out for individuals. So, this Robot Commands in manual mode using a Smartphone, Wireless cam for Real-time Broadcast, PIR and Metal detection sensors, GPS \& GSM modules, ultrasonic sensor, Gas \& Fire sensor, temperature and humidity sensor, Acid gun, RFID and Relay switch, In this wireless sensor networks (WSN) is an Encrypted Network and infrastructure-less wireless networks to Screening physical or natural conditions, such as vibrations, pressures, movement, or toxins and to agreeably transmit their information retrieved by using IOT Server.
\end{abstract}

Keywords: Passive Infra-Red (PIR), GPS, GSM, Radio frequency identification (RFID), wireless sensor networks (WSN), IOT.

\section{Introduction}

Recent sensational occasions such as the seismic tremors in Indonesia and japan or the numerous surges in Bangladesh had appeared that neighborhood respectful specialists and crisis administrations have inconveniences in satisfactorily overseeing emergency. Subsequently at any fiasco, to begin with 48 hours is pivotal for protecting victims. Fire, police department personnel work force and paramedics are being sent to play down the misfortune of resources, for diminishing misfortunes of victims and for getting exact data of the circumstance, a mechanical system can be utilized and can be adjusted concurring to desires of the circumstances.

\section{Literature Survey}

A low power wireless Miniature robot a seen from nowadays to very next day shows up cutting edge contraptions \& weapons are utilized by equipped forces for decreasing such peril for their life and to conquer their foes. Within this progression of modern innovation, so that it majorly depends on the forefront weapons or state of the craftsmanship systems being utilized [1-3]. Front line assistive bot that can be characterized as a bot that ousts the mines in battle all on its purpose and could be utilized for surveillance purposes on the foes.

\footnotetext{
${ }^{1} \mathrm{~S}$ Abiraj, Department of Electronics and Communication Engineering' Vel Tech High Tech Dr Rangarajan Dr Sakunthala Engineering College, Chennai, TN, India, E-mail: abiraj.ece17@velhightech.com.
} 
Those bots are really controlled remotely. The versatile secure robot comprises of four section that is specifically the Sensors, Micro-controller, Camera, Motor driver and Transmission unit. [4-6]

\section{Proposed System}

The Proposed system of our Bot consist of an operating system, in which we utilized Arduino UNO board, from now on Embedded C programming is utilized for commanding and to communicate with bot. The flow chart in Figure. 1

\subsection{Prototype Explanation}

The Layout is appeared underneath within the Figure. 2 portrays complete robotic prototype which outlines the Function of the whole prototype. In this Venture Microcontroller we utilized is Arduino Uno created by Arduino.cc.it is an open- source contraptions arrange basically based on Automatic voltage regulator (AVR) microcontroller Atmega328. Arduino Nano might moreover be a small, total, and breadboard-friendly board upheld the ATmega328. Passive infrared Ray (PIR) sensor is utilized to detect the development of alive victims within 6 to $7 \mathrm{~m}$ region.

An ultrasonic sensor may be a sensor that measures the space of a target address by transmitting waves that changes over the reflected sound into an electrical pulse. Metal Sensor identifies the metallic question which is display following to their dynamic side. This sensor works beneath the electrical foremost of inductance where a fluctuating current actuates a voltage (EMF) amid a target object. Gas sensors (too known as gas detectors) are electronic devices that distinguish and distinguish distinctive sorts of gasses. They are commonly utilized to distinguish poisonous or unstable gasses and degree gas concentration. A Humidity \& Temperature sensor (or hygrometer) resources, measures and reports both dampness \& examine the temperature. ESP32CAM may be a low fetched advancement Wi-Fi cam. This permits the IP cam for making ventures for live video spilling with diverse resolution. The ESP8266 is a Wireless Module can be a self-contained SOC (system on chip) with arranges TCP/IP tradition stack that can provide any microcontroller get to your wireless network. GPS (global positioning system) is utilized for pursing the arrange at an occurrence. GPS get the information from the satellite and save within its memory, at whatever point the information required is been transmitted by micro controller to base station. It may be a scaled down GSM (Global System for Mobile Communications) modem to achieve nearly anything a standard smart phone can, text messages through SMS, it can get or make phone calls, interfacing to network through GPRS (General Packet Radio Service). EM18 is a RFID reader which is utilized to read RFID (Radio-frequency identification) labels of frequency $125 \mathrm{kHz}$. After reading labels, it transmits unique ID serially to the PC.NRF is a transceiver module which implies that it can both send and get the information. The motor driver is utilized as an enhancer, to open up the supply voltage to the motors. Motor Driver L298N Module is a high-power motor driver module for driving stepper and DC Motors. which can be controlled in both clockwise and anticlockwise heading. The IOT server is utilized by establishing the connection between the robot and the user which can be controlled in both clockwise and anticlockwise heading. The IOT server is utilized by establishing the connection between the robot and the user. The electrically operated Relay switch comprises of input terminals for a single or different control signals and consist of 
working contact terminals. The robot is equipped with is Acid gun is actuated in case the suspicious human is unrecognized. A cellular smartphone phone is created with mobile OS which incorporates a few highlights of a PC operating system. This Smartphone has a numerous highlight such as, Internet, GPS and media player it acts like a personal digital assistant.

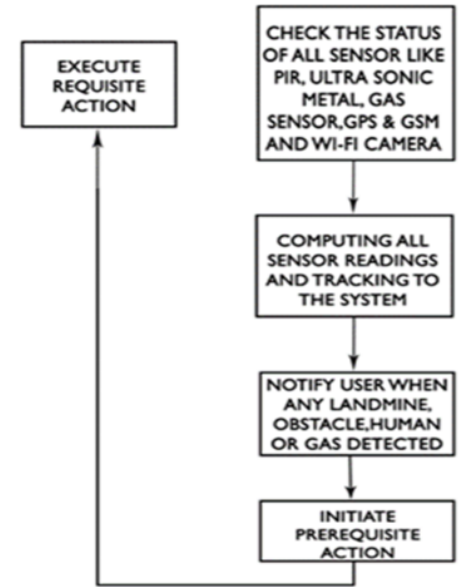

Figure 1. The Proposed system flow chart of the system

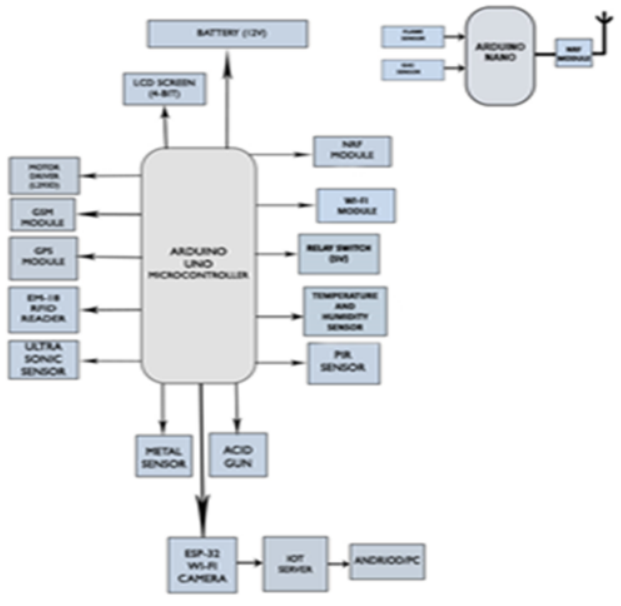

Figure 2. Layout of the proposed system

\subsection{Software Description}

The Flow Chart of Software description is shown in the Figure. 3 and the following programming tools are linked with the prototype and programed systems 1 . Arduino IDE 2. Embedded C. The Arduino IDE is a computer program which combines a program editing, Robotization tools, and debugging to build unique programmer for program headway. With a single user interface, Arduino IDE oversees all perspectives of embedded device advancement. The $\mathrm{C}$ Standards committee made Embedded $\mathrm{C}$ as a collection of language extensions for $\mathrm{C}$ programming to report issues and to provide medium-level get memory, and create a code creates format well for machines informational which needs negligible support to run-time time.

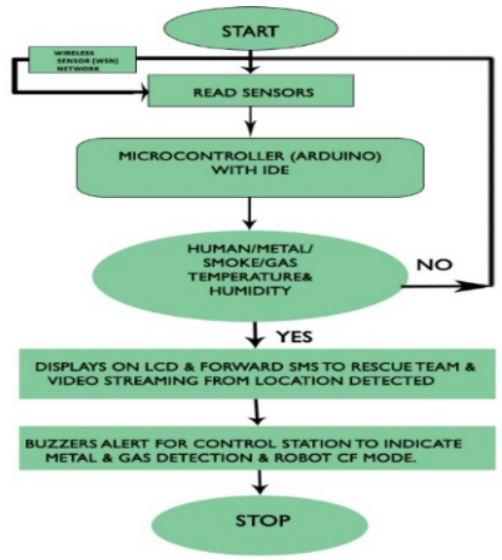

Figure 3. Software implementation flow chart

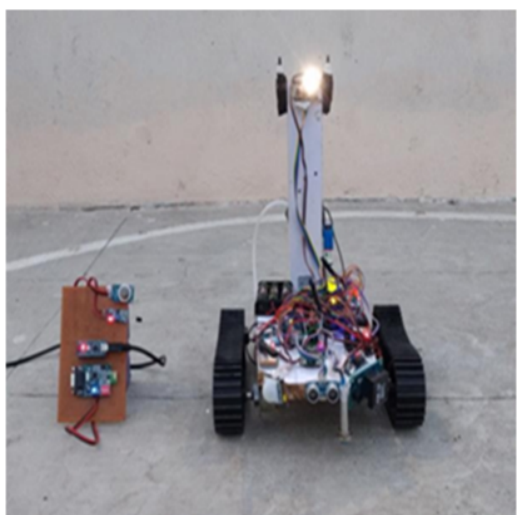

Figure 4. Final Prototype of Robot 


\section{Design Flow}

The Design Flow of this Robot is in hand-operated mode and it is shown in Figure. 5 In this Venture we utilized Portable device for informational is given to the controller which is associated through IP address, on the premise of given informational the bot wanders through like forward, in reverse, right, left, and stops in the event that any enlightening are not given it is appeared in Figure.6 We moreover utilized PIR sensor on the robot, at whatever point it faculties the movement inside the surmised area of $10 \mathrm{~m}$, the bot halt and informs user utilizing GSM that "Intruder detected". Wi-Fi cam will turn \& commanded by the user to testify around the object. The Acid shooter enacted in the event that necessary and on the off chance the Victim is suspicious, the Victim can be encountered on location. we used the Metal Sensor to identify landmines $\&$ explosives buried underneath land. bot halt \& area is followed by utilizing GPS. Coordinate values are followed \& gotten by the user. So that, the landmines \& Bombs is being dispensed. Ultrasonic Sensor, at whatever point a bot moving in a direction ultrasonic sensor transmits an ultrasonic pulse out at $40 \mathrm{kHz}$ which voyages through the air and in the event that there's a deterrent or obstacle, it'll recuperate through the sensor. In this Venture we used RFID tags used to read frequency $125 \mathrm{kHz}$. the tag comes in run of signal transmitted by the reader validate the card, in case an Invalid card is identified, at that point Caution SMS (short message service) is sent to user.

\section{Results and Discussion}

The robot is in manual mode by pressing the commanding button. A couple of detectors like gas and metal Sensors. which is executed for creating bot extra viable for distinguish harmful metals and gaseous and Information of this all is stored in IOT Server and its snapshot appeared below in Figure.7 Alert SMS is sent to the client on the off chance that any Victim or Metal is Detected is shown in Figure 8

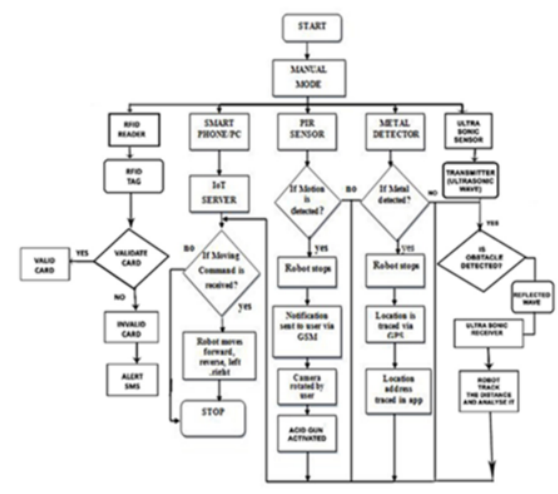

Figure 5. Flow chart of the robot operated in manual mode
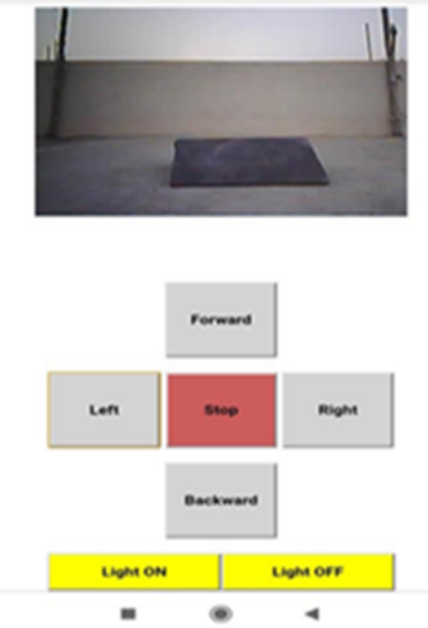

Figure 6. Operational Controlling of the Robot 

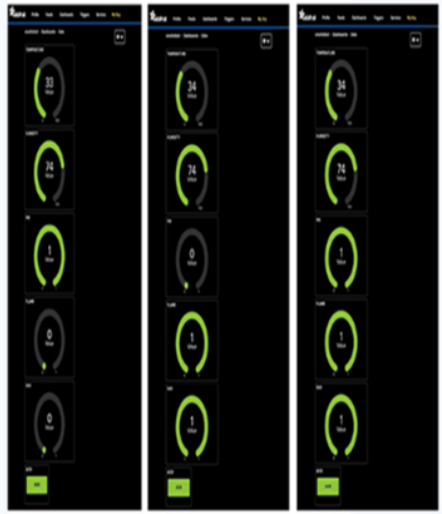

Figure 7. Snapshot of IoT Server

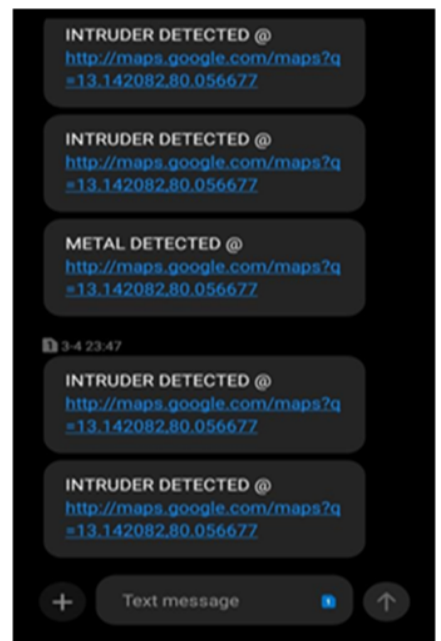

Figure 8. Snapshot of Alert SMS with Location.

\section{Conclusion and Future Scope}

Overall, this robot may be an exceedingly utilitarian gadget that decreases the workload on people in the midst of Disaster. Moreover, functions as a Surveillance vehicle to distinguish Armory items. In the midst of emergency \& especially in Metropolitan fiascos, this framework will be exceedingly valuable. The misfortune can be detected quickly and offer assistance comes to the victims. The advance innovation to be created out to surmount confinements of the Bot in such a way failure of this Bot will wander within tropical regions. For measures \& prototype of this Bot would be further diminished by utilizing inventive prototype and progressed fabric foe constructing Bot.

\section{References}

[1] Telkar, A. K., \& Gadgay, B. (2020). IoT Based Smart Multi Application Surveillance Robot. 2020 Second International Conference on Inventive Research in Computing Applications (ICIRCA), 931-935. https://doi.org/10.1109/ICIRCA48905.2020.9183289

[2] Anand Vijay, K. M., Purustut, S., Suhas, R., Pavan, C., \& Pradhan, P. (2018). A Live Human Being Detector in War Fields and Earthquake Location Using Robot with Camouflage Technology. 2018 3rd IEEE International Conference on Recent Trends in Electronics, Information \& Communication Technology (RTEICT), 2244-2249. https://doi.org/10.1109/ RTEICT42901.2018.9012601

[3] Aniruddha Prabhu, B. P., \& Hebbal, S. (2017). Small Unarmed Robot for Defense and Security:A Cost-Effective Approach Using Arduino Uno. 2017 2nd International Conference On Emerging Computation and Information Technologies (ICECIT), 1-6. https://doi.org/10.1109/ ICECIT.2017.8453325

[4] Joshi, S. A., Aravalli, G., Vidyashree, A. K., Ranade, S., \& Badami, S. S. (2017). Wireless controlled military combat robot system. 2017 2nd International Conference on Communication and Electronics Systems (ICCES), 712-715. https://doi.org/10.1109/CESYS.2017.8321173

[5] Murphy, R. R., Tadokoro, S., \& Kleiner, A. (2016). Disaster Robotics. In Springer Handbook of Robotics (pp. 1577-1604). Springer International Publishing. https://doi.org/10.1007/978-3-31932552-1_60 Aishwarya K Telkar and Prof.Baswaraj Gadgay, "IOT Based Smart Multi Application Surveillance Robot", Second International Conference on Inventive Research in Computing Applications (ICIRCA-2020), pp. 931, Sept 2020. 